\title{
A Class of Square Root and Division Free Algorithms and Architectures for QRD-Based Adaptive Signal Processing
}

\author{
E. N. Frantzeskakis, Member, IEEE, and K. J. R. Liu, Senior Member, IEEE
}

\begin{abstract}
The least squares (LS) minimization problem constitutes the core of many real-time signal processing problems, such as adaptive filtering, system identification and adaptive beamforming. Recently efficient implementations of the recursive least squares (RLS) algorithm and the constrained recursive least squares (CRLS) algorithm based on the numerically stable QR decomposition (QRD) have been of great interest. Several papers have proposed modifications to the rotation algorithm that circumvent the square root operations and minimize the number of divisions that are involved in the Givens: rotation. It has also been shown that all the known square root free algorithms are instances of one parametric algorithm. Recently, a square root free and division free algorithm has also been proposed. In this paper, we propose a family of square root and division free algorithms and examine its relationship with the square root free parametric family. We choose a specific instance for each one of the two parametric algorithms and make a comparative study of the systolic structures based on these two instances, as well as the standard Givens rotation. We consider the architectures for both the optimal residual computation and the optimal weight vector extraction. The dynamic range of the newly proposed algorithm for QRD-RLS optimal residual computation and the wordlength lower bounds that guarantee no overflow are presented. The numerical stability of the algorithm is also considered. A number of obscure points relevant to the realization of the QRD-RLS and the QRD-CRLS algorithms are clarified. Some systolic structures that are described in this paper are very promising, since they require less computational complexity (in various aspects) than the structures known to date and they make the VLSI implementation easier.
\end{abstract}

\section{INTRODUCTION}

$\mathbf{T}$ THE least squares (LS) minimization problem constitutes the core of many real-time signal processing problems, such as adaptive filtering, system identification and beamforming [6]. There are two common variations of the LS problem for adaptive signal processing:

1) Solve the minimization problem

$$
w(n)=\arg \min _{w(n)} \| \beta(n)\left\langle X(n) w(n)-y(n) ; \|^{2}\right.
$$

Manuscript received June 14, 1992; revised January 14, 1994. This work was supported in part by the ONR grant N00014-93-1-0566, the AASERT/ONR grant N00014-93-11028, and the NSF grant MIP9309506. The associate editor coordinating the review of this paper and approving it for publication was Prof. Monty Hayes.

The authors are with the Electrical Engineering Department and Institute for Systems Research, University of Maryland, College Park, MD 20742 USA.

IEEE Log Number 9403256. where $X(n)$ is a matrix of size $n \times p, w(n)$ is a vector of length $p, y(n)$ is a vector of length $n$ and $\beta(n)=\operatorname{diag}\left\{\beta^{n-1}, \beta^{n-2}, \cdots, 1\right\}, 0<\beta<1$, that is, $\beta$ is a forgetting factor.

2) Solve the minimization problem in (1) subject to the linear constraints

$$
c^{i T} w(n)=r^{i}, \quad i=1,2, \cdots, N
$$

where $c^{i}$ is a vector of length $p$ and $r^{i}$ is a scalar. In this paper, we consider only the special case of the minimum variance distortionless response (MVDR) beamforming problem [15] for which $y(n)=0$ for all $n$ and (1) is solved by subjecting to each linear constraint, i.e., there are $N$ linear-constrained LS problems.

There are two different pieces of information that may be required as the result of this minimization [6]:

1) The optimizing weight vector $w(n)$ and/or

2) the optimal residual at the time instant $n$

$$
e\left(t_{n}\right)=X\left(t_{n}\right) w(n)-y\left(t_{n}\right)
$$

where $X\left(t_{n}\right)$ is the last row of the matrix $X(n)$ and $y\left(t_{n}\right)$ is the last element of the vector $y(n)$.

Efficient implementations of the recursive least squares (RLS) algorithms and the constrained recursive least squares (CRLS) algorithms based on the QR decomposition (QRD) were first introduced by McWhirter [14], [15]. A comprehensive description of the algorithms and the architectural implementations of these algorithms is given in [6, chap.14]. It has been proved that the QRD-based algorithms have good numerical properties [6]. However, they are not very appropriate for VLSI implementation, because of the square root and the division operations that are involved in the Givens rotation and the backprintingsubstitution required for the case of weight extraction.

Several papers have proposed modifications in order to reduce the computational load involved in the original Givens rotation [2], [5], [4], [8]. These rotation-based algorithms are not rotations any more, since they do not exhibit the normalization property of the Givens rotation. Nevertheless, they can substitute for the Givens rotation as the building block of the QRD algorithm and thus they can be treated as rotation algorithms in a wider sense: 
Definition 1: A Givens-rotation-based algorithm that can be used as the building block of the QRD algorithm will be called a Rotation algorithm.

A number of square-root-free $\Re$ otations have appeared in the literature [2], [5], [8], [10]. It has been shown that a square-root-free and division-free $\Re$ otation does exist [4]. Recently, a parametric family of square-root-free $\Re$ otation algorithms was proposed in [8]; it was also shown that all the known square-root-free $\Re$ otation algorithms belong to this family, which is called the " $\mu \nu$-family." In this paper we will refer to the $\mu \nu$-family of $\Re$ otation algorithms with the name parametric $\mu \nu \Re$ Rotation. We will also say that a Rotation algorithm is a $\mu \nu \Re$ otation if this algorithm belongs to the $\mu \nu$ family. Several QRD-based algorithms have made use of these Rotation algorithms. McWhirter has been able to compute the optimal residual of the RLS algorithm without square root operations [14]. He also employed an argument for the similarity of the RLS and the CRLS algorithms to obtain a square-root-free computation for the optimal residual of the CRLS algorithm [15]. A fully-pipelined structure for weight extraction that circumvents the back-substitution divisions was also derived independently in [17] and in [19]. Finally, an algorithm for computing the RLS optimal residual based on the parametric $\mu \nu$ Rotation was derived in [8]

In this paper, we introduce a parametric family of squareroot-free and division-free Rotations. We will refer to this family of algorithms with the name parametric $\kappa \lambda$ Rotation. We will also say that a Rotation algorithm is a $\kappa \dot{\lambda} \Re$ otation if this algorithm is obtained by the parametric $\kappa \lambda \Re$ Rotation with a choice of specific values for the parameters $\kappa$ and $\lambda$. We employ the arguments in [8], [14], [15] and [17] in order to design novel architectures for the RLS and the CRLS algorithms that have less computation and circuit complexity. Some systolic structures that are described here are very promising, since they require the minimum computational complexity (in various aspects) known to date, and they can be easily implemented in VLSI.

In Section II, we introduce the parametric $\kappa \lambda$ ケotation. In Section III, we derive the RLS algorithms that are based on the parametric $\kappa \lambda$ 凡otation and we consider the architectural implementations for a specific $\kappa \lambda \Re$ Rotation. In Section IV, we follow the same procedure for the CRLS algorithms. In Section $\mathrm{V}$, we address the issues of dynamic range, lower bounds for the wordlength, stability and error bounds. We conclude with Section VI. In the Appendix we give the proofs of some lemmas that are stated in the course of the paper.

\section{Square Root and Division Free Algorithms}

In this section, we introduce a new parametric family of Givens-rotation-based algorithms that require neither square root nor division operations. This modification to the Givens rotation provides a better insight on the computational complexity optimization issues of the $\mathrm{QR}$ decomposition and makes the VLSI implementation easier.

\section{A. The Parametric $\kappa \lambda \Re$ Rotation}

The standard Givens rotation operates (for real-valued data) as follows:

$$
\left[\begin{array}{cccc}
r_{1}^{\prime} & r_{2}^{\prime} & \cdots & r_{m}^{\prime} \\
0 & x_{2}^{\prime} & \cdots & x_{m}^{\prime}
\end{array}\right]=\left[\begin{array}{cc}
c & s \\
-s & c
\end{array}\right]\left[\begin{array}{cccc}
\beta r_{1} & \beta r_{2} & \cdots & \beta r_{m} \\
x_{1} & x_{2} & \cdots & x_{m}
\end{array}\right]
$$

where

$$
\begin{array}{rlrl}
c & =\frac{\beta r_{1}}{\sqrt{\beta^{2} r_{1}^{2}+x_{1}^{2}}}, & s=\frac{x_{1}}{\sqrt{\beta^{2} r_{1}^{2}+x_{1}^{2}}} \\
r_{1}^{\prime} & =\sqrt{\beta^{2} r_{1}^{2}+x_{1}^{2}} \\
r_{j}^{\prime} & =c \beta r_{j}+s x_{j}, \quad j=1,2, \cdots, m \\
x_{j}^{\prime} & =-s \beta r_{j}+c x_{j}, \quad j=2,3, \cdots, m .
\end{array}
$$

We introduce the following data transformation

$$
\begin{aligned}
& r_{j}=\frac{1}{\sqrt{l_{a}}} a_{j}, \quad x_{j}=\frac{1}{\sqrt{l_{b}}} b_{j}, \quad r_{j}^{\prime}=\frac{1}{\sqrt{l_{a}^{\prime}}} a_{j}^{\prime}, \\
& j=1,2, \cdots, m \\
& x_{j}^{\prime}=\frac{1}{\sqrt{l_{b}^{\prime}}} b_{j}^{\prime}, \quad j=2,3, \cdots, m .
\end{aligned}
$$

We seek the square root and division-free expressions for the transformed data $a_{j}^{\prime}, j=1,2, \cdots, m, b_{j}^{\prime}, j=2,3, \cdots, m$, in (6) and solving for $a_{1}^{\prime}$, we get

$$
a_{1}^{\prime}=\sqrt{\frac{l_{a}^{\prime}}{l_{a} l_{b}}\left(l_{b} \beta^{2} a_{1}^{2}+l_{a} b_{1}^{2}\right)} .
$$

By substituting (5) and (9) in (7) and (8) and solving for $a_{j}^{\prime}$ and $b_{j}^{\prime}$, we get

$$
\begin{aligned}
a_{j}^{\prime} & =\frac{l_{b} \beta^{2} a_{1} a_{j}+l_{a} b_{1} b_{j}}{\sqrt{l_{a} l_{b}\left(l_{b} \beta^{2} a_{1}^{2}+l_{a} b_{1}^{2}\right) / l_{a}^{\prime}}} \quad \text { and } \\
b_{j}^{\prime} & =\frac{-b_{1} \beta a_{j}+\beta a_{1} b_{j}}{\sqrt{\left(l_{b} \beta^{2} a_{1}^{2}+l_{a} b_{1}^{2}\right) / l_{b}^{\prime}}} \quad j=2,3, \cdots, m .
\end{aligned}
$$

We will let $l_{a}^{\prime}$ and $l_{b}^{\prime}$ be equal to

$$
l_{a}^{\prime}=l_{a} l_{b}\left(l_{b} \beta^{2} a_{1}^{2}+l_{a} b_{1}^{2}\right) \kappa^{2}, \quad l_{b}^{\prime}=\left(l_{b} \beta^{2} a_{1}^{2}+l_{a} b_{1}^{2}\right) \lambda^{2}
$$

where $\kappa$ and $\lambda$ are two parameters. By substituting (12) in (10)-(11) we obtain the expressions

$$
\begin{aligned}
& a_{1}^{\prime}=\kappa\left(l_{b} \beta^{2} a_{1}^{2}+l_{a} b_{1}^{2}\right) \\
& a_{j}^{\prime}=\kappa\left(l_{b} \beta^{2} a_{1} a_{j}+l_{a} b_{1} b_{j}\right), \quad j=2,3, \cdots, m \text { and } \\
& b_{j}^{\prime}=\lambda \beta\left(-b_{1} a_{j}+a_{1} b_{j}\right), \quad j=2,3, \cdots, m .
\end{aligned}
$$

If the evaluation of the parameters $\kappa$ and $\lambda$ does not involve any square root or division operations, the update equations (12)-(15) will be square root and division-free. In other words, every such choice of the parameters $\kappa$ and $\lambda$ specifies a square root and division-free Rotation algorithm.

Definition 2: Equations (12)-(15) specify the parametric $\kappa \lambda \Re$ Rotation algorithm. Furthermore, a ßotation algorithm will be called a $\kappa \lambda$ 凡otation if it is specified by (12)-(15) for specific square-root-free and division-free expressions of the parameters $\kappa$ and $\lambda$.

One can easily verify that the only one square root and division-free Rotation in the literature to date [4] is a $\kappa \lambda$ $\Re$ otation and is obtained by choosing $\kappa=\lambda=1$. 


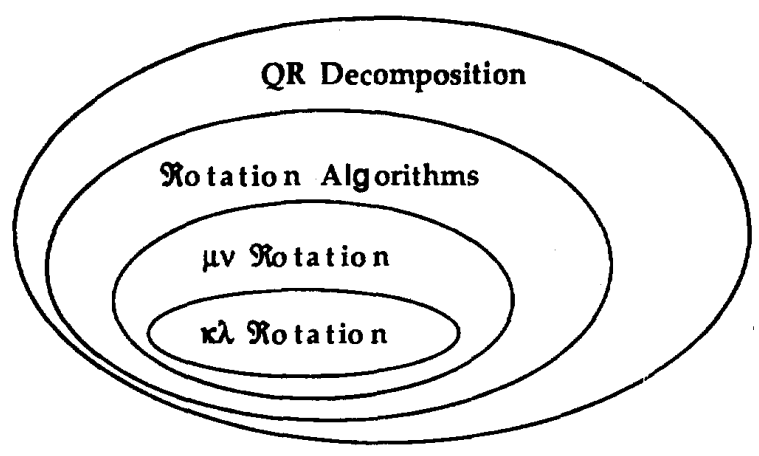

Fig. 1. The relations among the classes of algorithms based on $Q R$ decomposition, a Rotation algorithm, a $\mu \nu \Re$ otation, and a $\kappa \lambda \Re$ otation.

\section{B. The Relation between the Parametric $\kappa \lambda$} and the Parametric $\mu \nu$ Rotation

Let

$$
k_{a}=\frac{1}{l_{a}}, \quad k_{b}=\frac{1}{l_{b}}, \quad k_{a}^{\prime}=\frac{1}{l_{a}^{\prime}}, \quad k_{b}^{\prime}=\frac{1}{\frac{1}{i^{\prime}} .}
$$

We can express $k_{a}^{\prime}$ and $k_{b}^{\prime}$ in terms of $k_{a}$ and $k_{b}$ as follows [8]

$$
k_{a}^{\prime}=\left(k_{a} \beta^{2} a_{1}^{2}+k_{b} b_{1}^{2}\right) / \mu^{2}, \quad k_{b}^{\prime}=\frac{k_{a} k_{b}}{\mu^{2} \nu^{2}} \frac{1}{k_{a}^{\prime}} .
$$

If we substitute (16) and (17) in (12) and solve for $\mu$ and $\nu$ we obtain

$$
\mu=\frac{\kappa\left(k_{a} \beta^{2} a_{1}^{2}+k_{b} b_{1}^{2}\right)}{k_{a} k_{b}}, \quad \nu=\lambda .
$$

The above provides a proof for the following Lemrna:

Lemma 2.1: For each square root and division-free pair of parameters $(\kappa, \lambda)$ that specifies a $\kappa \lambda$ Æotation algorithm $A 1$, we can find square-root-free parameters $(\mu(\kappa), \nu(\lambda)$; with two properties: first, the pair $(\mu(\kappa), \nu(\lambda))$ specifies a $\mu \nu$ Rotation algorithm $A 2$ and second, both $A 1$ and $A 2$ are mathematically equivalent ${ }^{1}$.

Consequently, the set of $\kappa \lambda$ Rotation algorithmıs can be thought of as a subset of the set of the $\mu \nu$ 凡otations. Furthermore, (18) provides a means of mapping a $\kappa \lambda$ Rotation onto a $\mu \nu \Re$ otation. For example, one can verify that the square root and division-free algorithm in [4] is a $\mu \nu$ Rotation and is obtained for

$$
\mu=\frac{k_{a} \beta^{2} a_{1}^{2}+k_{b} b_{1}^{2}}{k_{a} k_{b}}, \quad \nu=1 .
$$

In Fig. 1, we draw a graph that summarizes the relations among the classes of algorithms based on QR deconposition, a $\Re$ otation algorithm, a $\mu \nu \Re$ otation and a $\kappa \lambda \Re$ Rotalion.

\section{RLS ALGORITHM AND ARCHITECTURE}

In this section, we consider the $\kappa \lambda$ Rotation for optimal residual and weight extraction using systolic array implementation. Detailed comparisons with existing approaches are presented.

${ }^{1}$ They evaluate logically equivalent equations.

\section{A. A Novel Fast Algorithm for the RLS Optimal Residual Computation}

The QR-decomposition of the data at time instant $n$ is as follows

$$
\left[\begin{array}{cc}
R(n) & u(n) \\
0^{T} & v\left(t_{n}\right)
\end{array}\right]=T(n)\left[\begin{array}{cc}
\beta R(n-1) & \beta u(n-1) \\
X\left(t_{n}\right) & y\left(t_{n}\right)
\end{array}\right]
$$

where $T(n)$ is a unitary matrix of size $(p+1) \times(p+1)$ that performs a sequence of $p$ Givens rotations. This can be written symbolically as

$$
\begin{aligned}
& {\left[\begin{array}{cc}
L(n-1)^{-\frac{1}{2}} & 0 \\
0^{T} & l_{q}(n)^{-\frac{1}{2}}
\end{array}\right]\left[\begin{array}{cc}
\beta \bar{R}(n-1) & \beta \bar{u}(n-1) \\
\bar{X}\left(t_{n}\right) & \bar{y}\left(t_{n}\right)
\end{array}\right]} \\
& \stackrel{T(n)}{\longrightarrow}\left[\begin{array}{cc}
L(n)^{-\frac{1}{2}} & 0 \\
0^{T} & l_{q}(n+1)^{-\frac{1}{2}}
\end{array}\right]\left[\begin{array}{cc}
\bar{R}(n) & \bar{u}(n) \\
0^{T} & b_{p+1}^{(p)}
\end{array}\right]
\end{aligned}
$$

where

$$
\begin{aligned}
L(\cdot)^{-\frac{1}{2}} \bar{R}(\cdot) & =R(\cdot), & L(\cdot)^{-\frac{1}{2}} \bar{u}(\cdot)=u(\cdot), \\
l_{q}(n)^{-\frac{1}{2}} \bar{X}\left(t_{n}\right) & =X\left(t_{n}\right), \quad & l_{q}(n)^{-\frac{1}{2}} \bar{y}\left(t_{n}\right)=y\left(t_{n}\right)
\end{aligned}
$$

and

$$
\begin{aligned}
& L(n-\mathbf{1})=\operatorname{diag}\left\{l_{1}, l_{2}, \cdots, l_{p}\right\} \\
& L(n)=\operatorname{diag}\left\{l_{1}^{\prime}, l_{2}^{\prime}, \cdots, l_{p}^{\prime}\right\}, \\
& \bar{R}(n-1)=\left[\begin{array}{cccc}
a_{11} & a_{12} & \cdots & a_{1 p} \\
& a_{22} & \cdots & a_{2 p} \\
& & \ddots & \vdots \\
& & & a_{p p}
\end{array}\right] \\
& \bar{R}(n)=\left[\begin{array}{cccc}
a_{11}^{\prime} & a_{12}^{\prime} & \cdots & a_{1 p}^{\prime} \\
& a_{22}^{\prime} & \cdots & a_{2 p}^{\prime} \\
& & \ddots & \vdots \\
& & & a_{p p}^{\prime}
\end{array}\right] \\
& \bar{u}(n-1)=\left[\begin{array}{llll}
a_{1, p+1} & a_{2, p+1} & \cdots & a_{p, p+1}
\end{array}\right]^{T} \\
& \bar{u}(n)=\left[\begin{array}{llll}
a_{1, p+1}^{\prime} & a_{2, p+1}^{\prime} & \cdots & a_{p, p+1}^{\prime}
\end{array}\right]^{T} \\
& {\left[\bar{X}\left(t_{n}\right) \bar{y}\left(t_{n}\right)\right]=\left[\begin{array}{llll}
b_{1} & b_{2} & \cdots & b_{p} \\
b_{p+1}
\end{array}\right] \text {. }}
\end{aligned}
$$

Equations (12)-(15) imply that the $i$ th $\Re$ otation is specified as follows

$$
\begin{gathered}
l_{i}^{\prime}=l_{i} l_{q}^{(i-1)}\left(l_{q}^{(i-1)} \beta^{2} a_{i i}^{2}+l_{i} b_{i}^{(i-1)^{2}}\right) \kappa_{i}^{2} \\
l_{q}^{(i)}=\left(l_{q}^{(i-1)} \beta^{2} a_{i i}^{2}+l_{i} b_{i}^{(i-1)^{2}}\right) \lambda_{i}^{2} \\
a_{i j}^{\prime}=\kappa_{i}\left(l_{q}^{(i-1)} \beta^{2} a_{i i} a_{i j}+l_{i} b_{i}^{(i-1)} b_{j}^{(i-1)}\right), \\
j=i, i+1, \cdots, p+1 \\
b_{j}^{(i)}=\lambda_{i} \beta\left(-b_{i}^{(i-1)} a_{i j}+a_{i i} b_{j}^{(i-1)}\right), \\
j=i+1, i+2, \cdots, p+1
\end{gathered}
$$

where $i=1,2, \cdots, p, b_{j}^{(0)}=b_{j}, j=1, \cdots, p+1$ and $l_{q}^{(0)}=l_{q}$. For the optimal residual we have: 

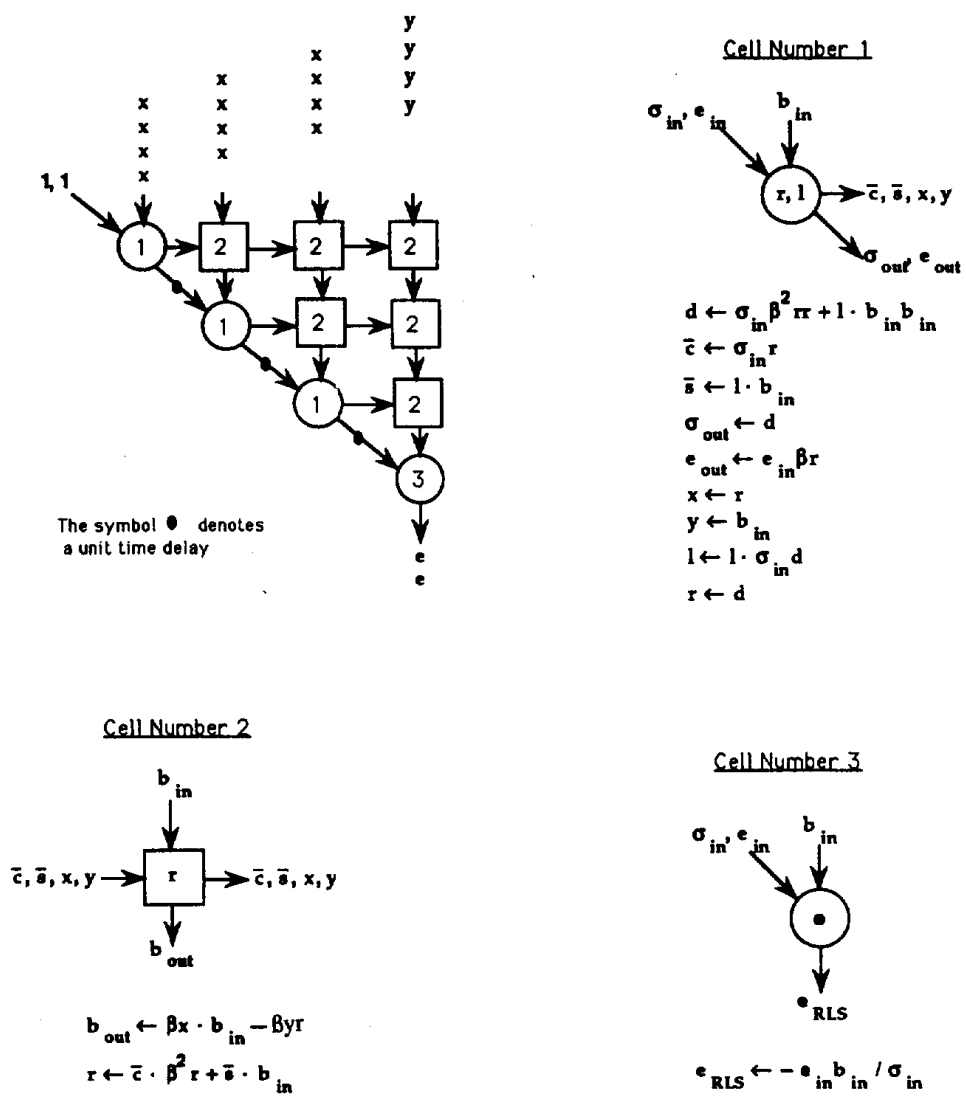

Fig. 2. $S 1.1$ : Systolic array that computes the RLS optimal residual. It implements the algorithm that is based on the $\kappa \lambda$ Rotation for which $\kappa=\lambda=1$.

Lemma 3.I: If the parametric $\kappa \lambda$ Rotation is used in the QRD-RLS algorithm, the optimal residual is given by the expression

$$
e_{R L S}\left(t_{n}\right)=-\left(\prod_{i=1}^{p-1} \lambda_{i} \beta a_{i i}\right) \frac{\kappa_{p} \beta a_{p p}}{\lambda_{p} a_{p p}^{\prime}} b_{p+1}^{(p)} \sqrt{l_{q}}
$$

The proof is given in the Appendix.

Here, $l_{q}$ is a free variable. If we choose $l_{q}=1$, we can avoid the square root operation. We can see that for a recursive computation of (26) only one division operation is needed at the last step of the recursion. This compares very favorably with the square root free fast algorithms that require one division for every recursion step, as well as with the original approach (63), which involves one division and one square root operation for every recursion step.

The division operation in (26) cannot be avoided by proper choice of expressions for the parameters $\kappa$ and $\lambda$. This is restated by the following Lemma, which is proved in the Appendix:

Lemma 3.2: If a $\kappa \lambda \Re$ Rotation is used, the RLS optimal residual evaluation will require at least one division evaluation.
Note that the proper choice of the expression for the parameter $\lambda_{p}$, along with the rest of the parameters, is an open question, since the minimization of the multiplication operations, as well as communication and stability issues have to be considered.

\section{B. A Systolic Architecture for the Optimal RLS Residual Evaluation}

McWhirter has used a systolic architecture for the implementation of the QR decomposition [14]. This architecture is modified, so that equations (22)-(26) be evaluated for the special case of $\kappa_{i}=\lambda_{i}=1, i=1,2, \cdots, p$ and $l_{q}=1$. The systolic array, as well as the memory and the communication links of its components, are depicted in Fig. $2^{2}$. The boundary cells (cell number 1) are responsible for evaluating (22) and (23), as well as the coefficients $\bar{c}_{i}=l_{q}^{(i-1)} a_{i i}$ and $\bar{s}_{i}=$ $l_{i} b_{i}^{(i-1)}$ and the partial products $e_{i}=\prod_{j=1}^{i}\left(\beta a_{j j}\right)$. The internal cells (cell number 2 ) are responsible for evaluating (24) and (25). Finally, the output cell (cell number 3 ) evaluates (26). The functionality of each one of the cells is described in Fig. 2. We will call this systolic array $S 1.1$.

\footnotetext{
${ }^{2}$ Note the aliases $l_{q}^{(i-1)} \equiv \sigma_{i n}, l_{q}^{(i)} \equiv \sigma_{\text {out }}, l_{i} \equiv l, a_{i j} \equiv r, b_{j}^{(i-1)} \equiv$ $b_{\text {in }}, b_{j}^{(i)} \equiv b_{\text {out }}, e_{i-1} \equiv e_{\text {in }}, e_{i} \equiv e_{\text {out }}$.
} 
TABLE I

RLS RESIDUAL COMPUTATIONAL COMPLEXITY

\begin{tabular}{|c|ccc|ccc|ccc|}
\hline & \multicolumn{3}{|c|}{$S 1.1: \kappa \lambda$} & \multicolumn{3}{c|}{$S 1.2: \mu \nu$} & \multicolumn{3}{c|}{$S 1.3:$ Giver rotation } \\
\hline cell & 1 & 2 & 3 & 1 & 2 & 3 & 1 & 2 & 3 \\
\hline number of & $p$ & $\frac{p(p+1)}{2}$ & 1 & $p$ & $\frac{p(p+1)}{2}$ & 1 & $p$ & $\frac{p(p+1)}{2}$ & 1 \\
\hline sq.rt & - & - & - & - & - & - & 1 & - & - \\
div. & - & - & 1 & 1 & - & - & 1 & - & - \\
mult. & 9 & 4 & 1 & 5 & 3 & 1 & 4 & 4 & 1 \\
i/o & 9 & 10 & 4 & 6 & 8 & 3 & 5 & 6 & 3 \\
\hline
\end{tabular}

On Table I, we collect some features of the systolic structure $S 1.1$ and the two structures, $S 1.2$ and $S 1.3$, in [1;] that are pertinent to the circuit complexity. The $S 1.2$ implements the square-root-free QRD-RLS algorithm with $\mu=\nu:=1$, while $S 1.3$ is the systolic implementation based on the original Givens rotation. In Table I, the complexity per processor cell and the number of required processor cells are indicated for each one of the three different cells ${ }^{3}$. One can easi y observe that $S 1.1$ requires only one division operator and no square root operator, $S 1.2$ requires $p$ division operators and no square root operator, while $S 1.3$ requires $p$ division and $p$ square root operators. This reduction of the complexity in terms of division and square root operators is penalized with the increase of the number of the multiplications and the communication links that are required.

Apart from the circuit complexity that is involved in the implementation of the systolic structures, another feature of the computational complexity is the number of operations-percycle. This number determines the minimum requ red delay between two consecutive sets of input data. For the structures $S 1.2$ and $S 1.3$ the boundary cell (cell number 1) constitutes the bottleneck of the computation and therefore it cetermines the operations-per-cycle that are shown on Table $r$. For the structure $S 1.1$ either the boundary cell or the output cell are the bottleneck of the computation.

\section{A Systolic Architecture for the Optimal RLS Weight Extraction}

Shepherd et al. [17] and Tang et al. [19] have independently shown that the optimal weight vector can be evaluated in a recursive way. More specifically, one can compute recursively the term $R^{-T}(n)$ by

$$
\left[\begin{array}{c}
R^{-T}(n) \\
\#
\end{array}\right]=T(n)\left[\begin{array}{c}
\frac{1}{\beta} R^{-T}(n-1) \\
0^{T}
\end{array}\right]
$$

and then use parallel multiplication for computing $u^{T}(n)$ by

$$
w^{T}(n)=u^{T}(n) R^{-T}(n) .
$$

The symbol \# denotes a term of no interest. The above algorithm can be implemented by a fully pipelinerl systolic array that can operate in two distinct modes, 0 ard 1 . The initialization phase consists of $2 p$ steps for each processor. During the first $p$ steps the processors operate in mode 0 in order to calculate a full rank matrix $R$. During the following $p$ steps, the processors operate in mode 1 in order to compute $R^{-T}$, by performing a task equivalent to forward substitution.

\footnotetext{
${ }^{3}$ The multiplications with the constants $\beta$ and $3^{2}$ are not encountered.
}

After the initialization phase the processors operate in mode 0 . In [17] one can find the systolic array implementations based both on the original Givens rotation and the Gentleman's variation of the square-root-free Rotation, that is, the $\mu \nu$ Rotation for $\mu=\nu=1$. We will call these two structures $S 2.3$ and $S 2.2$, respectively.

In Fig. 3, we present the systolic structure $S 2.1$ based on the $\kappa \lambda$ Rotation with $\kappa_{i}=\lambda_{i}=1, i=1,2, \cdots, p$. This is a square-root-free and division-free implementation. The boundary cells (cell number 1) are slightly simpler than the corresponding ones of the array $S 1.1$. More specifically, they do not compute the partial products $e_{i}$. The internal cells (cell number 2), that compute the elements of the matrix $R$, are identical to the corresponding ones of the array $S 1.1$. The cells that are responsible for computing the vector $u$ (cell number 3) differ from the other internal cells only in the fact that they communicate their memory value with their right neighbors. The latter (cell number 4) are responsible for evaluating (28) and (27). The functionality of the processing cells, as well as their communication links and their memory contents, are given in Fig. 3. The mode of operation of each cell is controlled by the mode bit provided from the input. For a more detailed description of the operation of the mode bit one can see [15] and [17].

On Tables II and V, we collect some computational complexity metrics for the systolic arrays $S 2.1, S 2.2$ and $S 2.3$, when they operate in mode $0^{4}$. The conclusions we can draw are similar to the ones we had for the circuits that calculate the optimal residual: the square root operations and the division operations can be eliminated with the cost of an increased number of multiplication operations and communication links. We should also note that $S 2.1$ does require the implementation of division operators in the boundary cells, since these operators are used during the initialization phase. Nevertheless, after the initialization phase the circuit will not suffer from any time delay caused by division operations. The computational bottleneck of all three structures, $S 2.1, S 2.2$ and $S 2.3$, is the boundary cell, thus it determines the operations-per-cycle metric.

As a conclusion for the RLS architectures, we observe that the figures on Tables I, II, and V favor the architectures based on the $\kappa \lambda$ Rotation, $\kappa=\lambda=1$ versus the ones that are based on the $\mu \nu$ rotation with $\mu=\nu=1$ and the standard Givens rotation. This claim is clearly substantiated by the delay times on Table V, associated to the DSP implementation of the QRD-RLS algorithm. These delay times are calculated on the basis of the manufacturers benchmark speeds for floating point operations [stewart]. Due to the way of updating $R^{-1}$, such a weight extraction scheme will have a numerical stability problem if the weight vector at each time instant is required.

\section{CRLS Algorithm AND ARCHITECTURE}

The optimal weight vector $w^{i}(n)$ and the optimal residual $e_{C R L S}^{i}\left(t_{n}\right)$ that correspond to the $i$ th constraint vector $c^{i}$ are given by the expressions [15]

\footnotetext{
${ }^{4}$ The multiplications with the constants $3, \beta^{2}, 1 / \beta$ and $1 / 3^{2}$, as well as
} the communication links that drive the mode bit, are not encountered. 

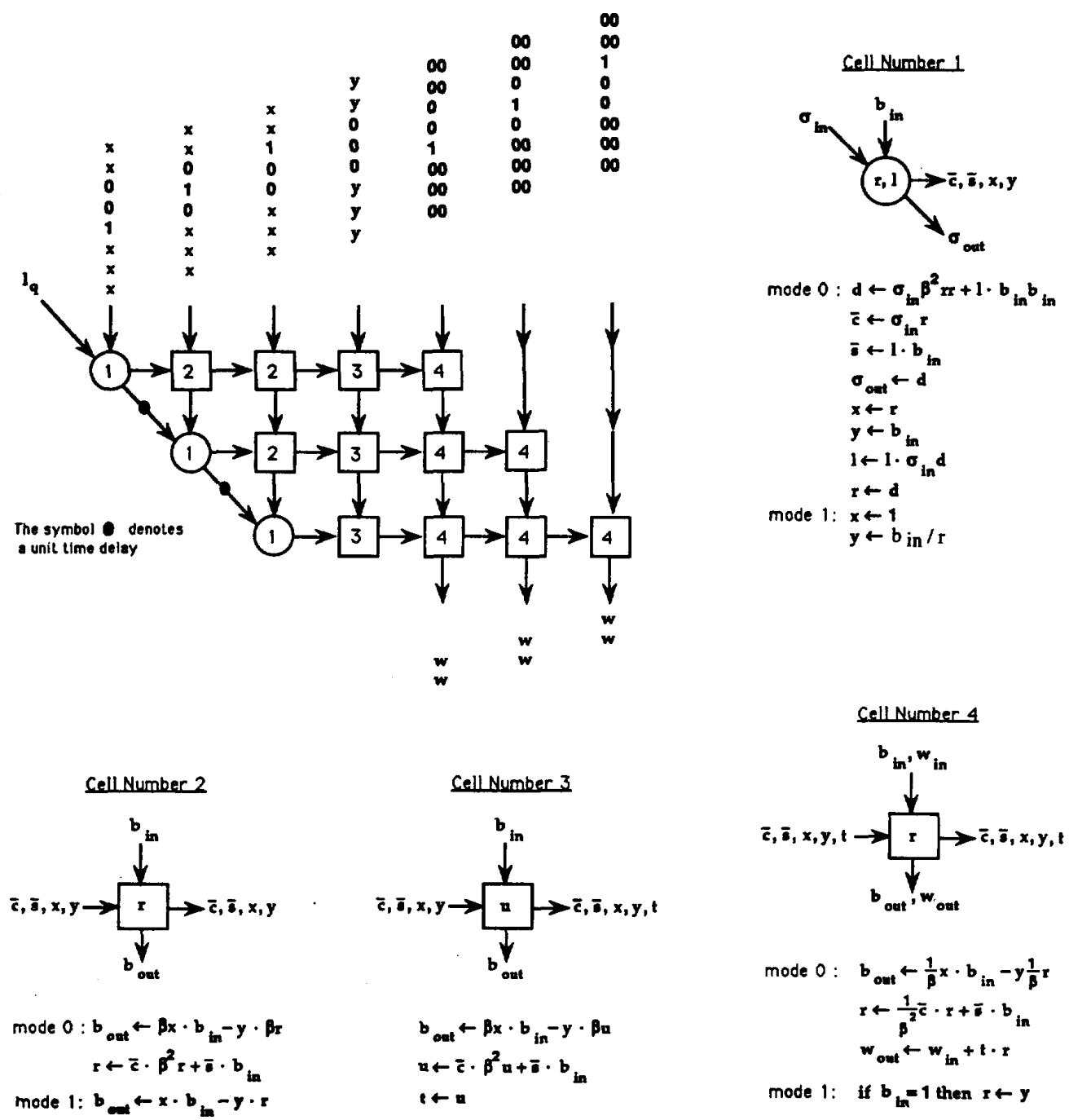

Fig. 3. $S 2.1$ : Systolic array that computes the RLS optimal weight vector. It implements the algorithm that is based on the $\kappa \lambda \Re$ otation for which $\kappa=\lambda=1$.

TABLE II

RLS Weight EXרraction COMPUTATIONAL CompleXITy (MODE 0)

\begin{tabular}{|c|cccc|cccc|cccc|}
\hline & \multicolumn{4}{|c|}{$S 2.1: \kappa \lambda$} & \multicolumn{4}{c|}{$S 2.2: \mu \nu$} & \multicolumn{3}{c|}{$S 2.3:$ Givens rotation } \\
\hline cell & 1 & 2 & 3 & 4 & 1 & 2 & 3 & 4 & 1 & 2 & 3 & .4 \\
\hline number of & $p$ & $\frac{(p-1) p}{2}$ & $p$ & $\frac{p}{(p+1)}$ & $p$ & $\frac{(p-1) p}{2}$ & $p$ & $\frac{p(p+1)}{2}$ & $p$ & $\frac{(p-1) p}{2}$ & $p$ & $\frac{p(p+1)}{2}$ \\
\hline sq.rt & - & - & - & - & - & - & - & - & 1 & - & - & - \\
div. & - & - & - & - & 1 & - & - & - & 1 & - & - & - \\
mult. & 8 & 4 & 4 & 5 & 5 & 3 & 3 & 4 & 4 & 4 & 4 & 5 \\
i/o & 7 & 10 & 11 & 14 & 6 & 8 & 9 & 12 & 3 & 6 & 7 & 10 \\
\hline
\end{tabular}

$$
w^{i}(n)=\frac{r^{i}}{\left\|z^{i}(n)\right\|^{2}} R^{-1}(n) z^{i}(n)
$$

and

$$
e_{C R L S}^{i}\left(t_{n}\right)=\frac{r^{i}}{\left\|z^{i}(n)\right\|^{2}} \hat{e}_{C R L S}^{i}\left(t_{n}\right)
$$

where

$$
\hat{e}_{C R L S}^{i}\left(t_{n}\right)=X\left(t_{n}\right) R^{-1}(n) z^{i}(n) .
$$

The term $z^{i}(n)$ is defined as follows

$$
z^{i}(n)=R^{-T}(n) c^{i}
$$


and it is computed with the recursion [15]

$$
\left[\begin{array}{c}
z^{i}(n) \\
\#
\end{array}\right]=T(n)\left[\begin{array}{c}
\frac{1}{\beta} z^{i}(n-1) \\
0^{T}
\end{array}\right]
$$

where the symbol \# denotes a term of no intere it. In this section, we derive a variation of the recursion tha: is based

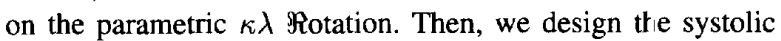
arrays that implement this recursion for $\kappa=\lambda=1$. We also make a comparison of these systolic structures with those based on the Givens rotation and the $\mu \nu$ Rotation introduced by Gentleman [6], [2], [15], [17].

From (32) and (21) we have $z^{i}(n)=\left(L(n)^{-1 / 2} \bar{h}^{\prime}(n)\right)^{-T} c^{i}$ and since $L(n)$ is a diagonal real valued matrix we get $z^{i}(n)=$ $L(n)^{1 / 2} \bar{R}(n)^{-T} c^{i}$, where $c^{i}$ is the constraint direct on. If we let

$$
\bar{z}^{i}(n)=L(n) \bar{R}(n)^{-T} c^{i}
$$

we obtain

$$
z^{i}(n)=L(n)^{-1 / 2} \bar{z}^{i}(n) .
$$

From (35) we get $\left\|z^{i}(n)\right\|^{2}=\bar{z}^{i^{T}}(n) L^{-1}(n) \bar{z}^{i}(n)$. Also, from (21) and (35) we get $R^{-1}(n) z^{i}(n)=\bar{R}^{-1}(n) \bar{z}^{i}(n)$. Consequently, from (29), (30), (31) we have

$$
e_{C R L S}^{i}(n)=\frac{r^{i}}{\bar{z}^{i T}(n) L^{-1}(n) \bar{z}^{i}(n)} \hat{e}_{C R L S}^{i}(n)
$$

and

$$
w^{i}(n)=\frac{r^{i}}{\bar{z}^{i T}(n) L^{-1}(n) \bar{z}^{i}(n)} \bar{R}^{-1}(n) \bar{z}^{i}(n)
$$

where

$$
\hat{e}_{C R L S}^{i}(n)=X(n) \bar{R}^{-1}(n) \bar{z}^{i}(n) .
$$

Because of the similarity of (31) with (38) and (29) with (37) we are able to use a variation of the systolic arrays that are based on the Givens rotation [15], [17] in order to evaluate (36)-(37).

\section{A. Systolic Architecture for the Optimal CRLS Residual Evaluation}

From (26) and (36), if $l_{q}=1$, we get the optimal residual

$e_{C R L S}^{i}(n)=-\frac{r^{i}}{\bar{z}^{i^{T}}(n) L^{-1}(n) \bar{z}^{i}(n)}\left(\prod_{j=1}^{p-1} \lambda_{j} a_{j j}\right) \frac{\kappa_{p}}{\lambda_{p}} \frac{a_{p p}}{a_{p p}^{\prime}} b_{p+1}^{(p)}$.

In Fig. 4 , we present the systolic array $S 3.1$, that evaluates the optimal residual for $\kappa_{j}=\lambda_{j}=1, j=1,2, \cdots, p$, and the number of constraints is $N=2$. This systolic: array is based on the design proposed by McWhirter [15]. I $\mid$ operates in two modes and is in a way very similar to the operation of the systolic structure $S 2.1$ (see Section III). The recursive equations for the data of the matrix $\bar{R}$ are given in (22)-(25). They are evaluated by the boundary cells (cell number 1) and the internal cells (cell number 2 ). These internal cells are identical to the ones of the array $S 2.1$. The boundary cells have a very important difference from the corresponding ones of $S 2.1$ : while they operate in mode 0 , they make use of their division operators in order to evaluate the elements of the diagonal matrix $L^{-1}(n)$, i.e., the quantities $1 / l_{i}, i=$ $1,2, \cdots, p$. These quantities are needed for the evaluation of the term $\bar{z}^{i^{T}}(n) L^{-1}(n) \bar{z}^{i}(n)$ in (39). The elements of the vectors $\bar{z}^{1}$ and $\bar{z}^{2}$ are updated by a variation of (24) and (25), for which the constant $\beta$ is replaced by $1 / \beta$. The two columns of the internal cells (cell number 3 ) are responsible for these computations. They initialize their memory value during the second phase of the initialization (mode 1) according to (34). While they operate in mode 0 , they are responsible for evaluating the partial sums

$$
\eta_{k}=\sum_{j=1}^{k}\left\|\bar{z}_{j}^{i}\right\|^{2} / l_{j}
$$

The output cells (cell number 4) are responsible for the final evaluation of the residual ${ }^{5}$.

McWhirter has designed the systolic arrays that evaluate the optimal residual, based on either the Givens rotation or the square-root-free variation that was introduced by Gentleman [2], [15]. We will call these systolic arrays $S 3.3$ and $S 3.2$, respectively. On Tables III and V we collect some computational complexity metrics for the systolic arrays $S 3.1, S 3.2$ and $S 3.3$, when they operate in mode $0^{6}$. We observe that the $\mu \nu \Re$ otation-based $S 3.2$, outperforms the $\kappa \lambda$ Rotation-based $S 3.1$. The two structures require the same number of division operators, while $S 3.2$ needs less multipliers and also it has less communication overhead.

\section{B. A Systolic Architecture for the Optimal CRLS Weight Vector Extraction}

In Fig. 5, we present the systolic array that evaluates (37) for $\kappa_{j}=\lambda_{j}=1, j=1,2, \cdots, p$ and the number of constraints equal to $N=2$. This systolic array operates in two modes, just as the arrays $S 2.1$ and $S 3.1 \mathrm{do}$. The boundary cell (cell number 1) is responsible for evaluating the diagonal elements of the matrices $R$ and $L$, the variable $l_{q}$, as well as all the coefficients that will be needed in the computations of the internal cells. In mode 0 its operation is almost identical to the operation of the boundary cell in $S 2.1$ (except for $t$ ), while in mode 1 it behaves like the corresponding cell of $S 3.1$. The internal cells in the left triangular part of the systolic structure (cell number 2) evaluate the nondiagonal elements of the matrix $R$ and they are identical to the corresponding cells of $S 3.1$. The remaining part of the systolic structure is a 2-layer array. The cells in the first column of each layer (cell number 3 ) are responsible for the calculation of the vector $z^{i}$ and the partial summations (40). They also communicate their memory values to their right neighbors. The latter (cell number 4 ) evaluate the elements of the matrix $R^{-T}$ and they are identical to the corresponding elements of $S 2.1$. The output elements (cell number 5) are responsible for the normalization of the weight vectors and they compute the final result.

\footnotetext{
${ }^{5}$ Note the alias $r^{i} \equiv \tau$.

${ }^{6}$ The multiplications with the constants $\beta, \beta^{2}, 1 / \beta$ and $1 / \beta^{2}$, as well as the communication links that drive the mode bit, are not encountered.
} 

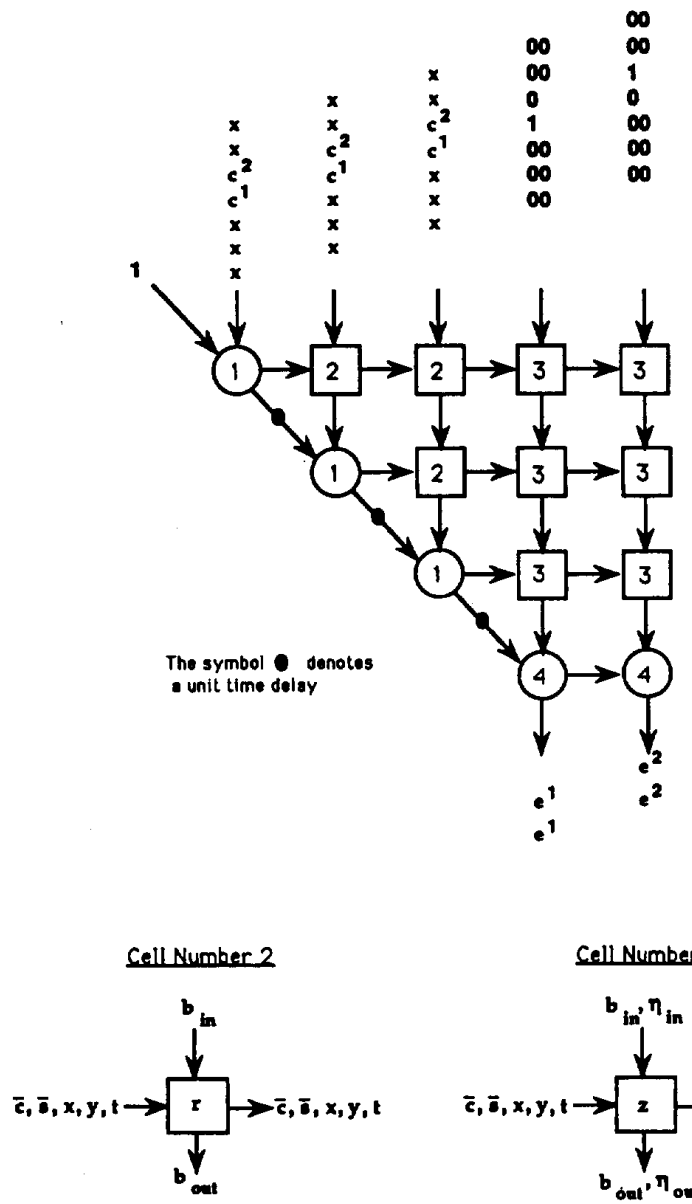

mode $0: b_{\text {out }} \leftarrow \beta x \cdot b_{\text {in }}-y \cdot \beta_{x}$

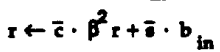

mode 1: $b_{\text {out }} \leftarrow x \cdot b_{\ln }-y \cdot r$
Cell Number 3

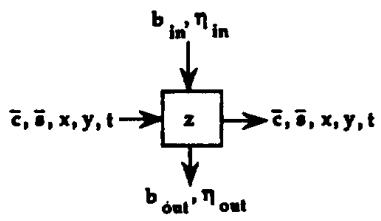

mode 0: $b_{\text {out }} \leftarrow \frac{1}{\beta} x \cdot b_{\text {in }}-y \frac{1}{\beta} z$

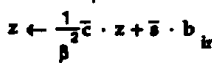

$\eta_{\text {out }} \leftarrow \eta_{\text {in }}+t \cdot 2 \cdot z$

mode 1: if $b_{1}=1$ then $z \leftarrow y \cdot t$

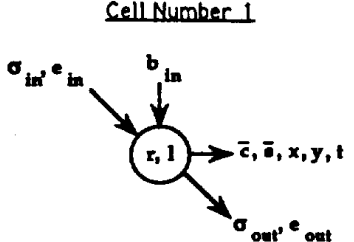

mode $0: d \leftarrow \sigma_{i n} \beta^{2} r+1 \cdot b_{\text {in }} b_{\text {in }}$

$\bar{c} \leftarrow \sigma_{\text {in }}{ }^{2}$

$a+1 \cdot b_{\text {in }}$

$\sigma_{0 a t} \leftarrow d$

$e_{\text {out }} \leftarrow e_{\ln }^{\beta x}$

$x \leftarrow x$

$y \leftarrow b_{\text {in }}$

$1 \leftarrow 1 \cdot \sigma_{\text {in }} d$

$x \leftarrow d$

$t \leftarrow|1|$

mode 1: $x \leftarrow 1$

$y \leftarrow b$ in $/ r$

Cell Number 4
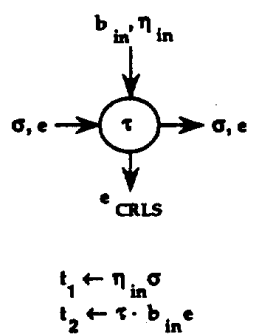

CRLs $\leftarrow-t_{2} / t_{1}$

Fig. 4. $S 3.1$ : Systolic array that computes the CRLS optimal residual. It implements the algorithm that is based on the $\kappa \lambda \Re$ otations for which $\kappa=\lambda=1$.

TABLE III

CRLS Optimal Residual Computational ComplextTy (MOde 0)

\begin{tabular}{|c|c|c|c|c|c|c|c|c|c|c|c|c|}
\hline & \multicolumn{4}{|c|}{$\overline{S 3.1:} \overline{\kappa \lambda}$} & \multicolumn{4}{|c|}{$S 3.2: \mu \nu$} & \multicolumn{4}{|c|}{$S 3.3$ : Givens rotation } \\
\hline cell & 1 & $\overline{2}$ & 3 & 4 & 1 & 2 & 3 & 4 & 1 & 2 & 3 & 4 \\
\hline number of & $p$ & $\frac{(p-1) p}{2}$ & $N p$ & $N$ & $p$ & $\frac{(p-1) p}{2}$ & $N p$ & $N$ & $p$ & $\frac{(p-1) p}{2}$ & $N p$ & $N$ \\
\hline sq.rt & - & - & - & - & $=$ & - & - & - & 1 & - & - & - \\
\hline div. & 1 & - & - & 1 & 1 & - & . & 1 & 1 & - & - & 1 \\
\hline mult. & 9 & 4 & 6 & 3 & 6 & 3 & 5 & 2 & 5 & 4 & 5 & 2 \\
\hline$i / o$ & 10 & 12 & 14 & 7 & 7 & 10 & 12 & 5 & 5 & 6 & 8 & 5 \\
\hline
\end{tabular}

Shepherd et al. [17] and Tang et al. [19] have designed systolic structures for the weight vector extraction based on the Givens rotation and the square-root-free $\Re$ otation of Gentleman [2]. We will call these two arrays $S 4.3$ and $S 4.2$, respectively. On Tables IV and V, we show the computational complexity metrics for the systolic arrays $S 4.1, S 4.2$ and $S 4.3$, when they operate in mode 0 . The observations we make are similar to the ones we have for the systolic arrays that evaluate the RLS weight vector (see Section III).

Note that each part of the 2-layer structure computes the terms relevant to one of the two constraints. In the same way, a problem with $N$ constraints will require an $N$-layer structure. With this arrangement of the multiple layers we obtain a unit time delay between the evaluation of the weight 


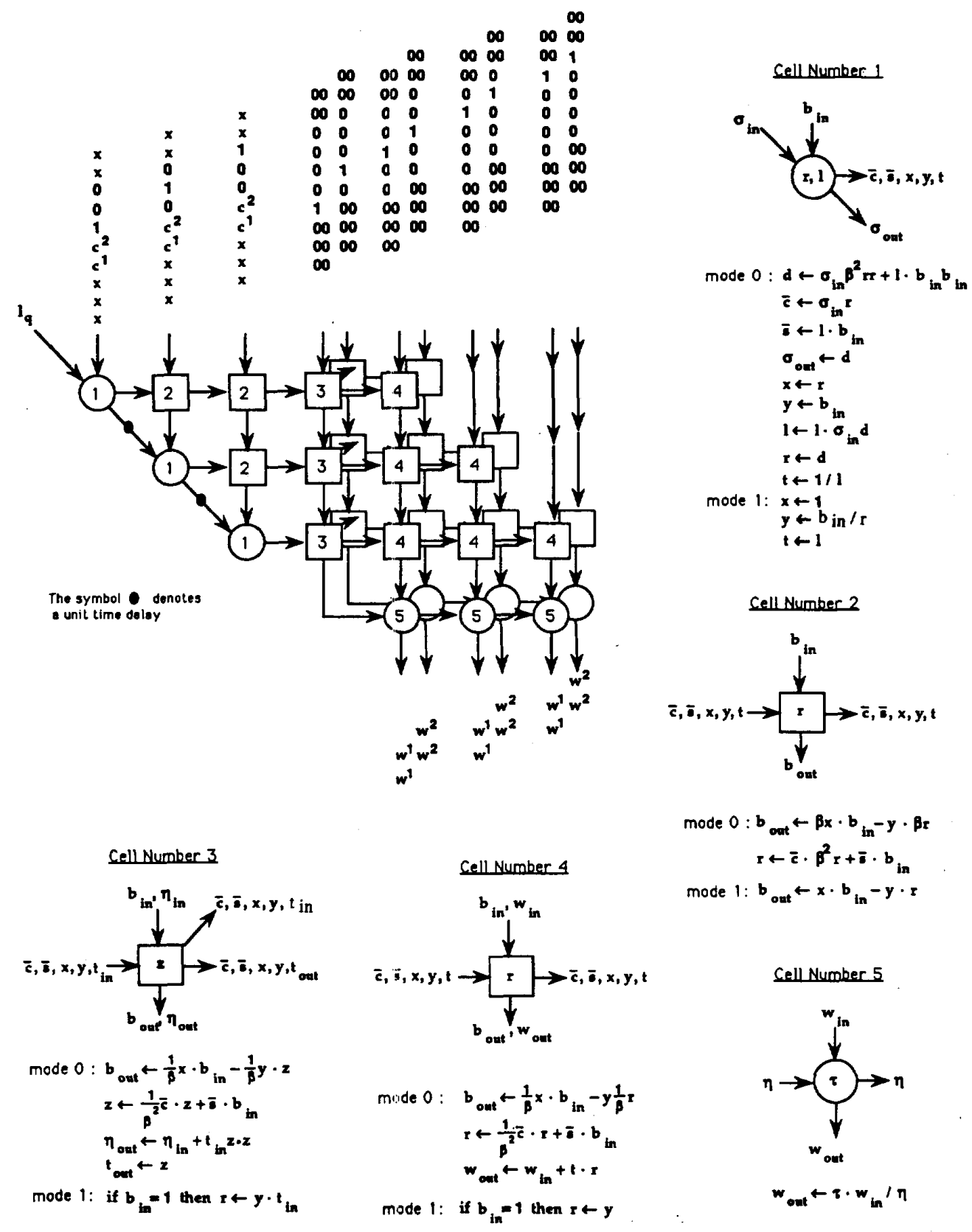

Fig. 5. S4.1 : Systolic array that computes the CRLS optimal weight vector. It implements the algorithm that is based on the $\kappa \lambda \Re$ $\Re$ otation for which $\kappa=\lambda=1$.

vectors for the different constraints. The price we have to pay is the global wiring for some of the communication links of cell 3. A different approach can also be considered: we may place the multiple layers side by side, one on the right of the other. In this way, not only the global wiring will be avoided, but also the number of communication links of cell 3 , will be considerably reduced. The price we will pay with this approach is a time delay of $p$ units between consequent evaluations of the weight vectors for different constraints.
As a conclusion for the CRLS architectures, we observe that the figures on Tables III, IV and V favor the architectures based on the $\mu \nu$ Rotation, $\mu=\nu=1$ versus the ones that are based on the $\kappa \lambda$ rotation with $\kappa=\lambda=1$.

\section{DYNAMIC RANGE, STABILITY, AND ERRoR BOUNDS}

Both the $\kappa \lambda$ and $\mu \nu$ Rotation algorithms enjoy computational complexity advantages compared to the standard Givens rotation with the cost of the denormalization of the latter. 
TABLE IV

CRLS Weight Vector EXTRACTION COMP COMPLEXITY (MODE 0)

\begin{tabular}{|c|c|c|c|c|c|c|c|c|c|c|}
\hline & \multicolumn{5}{|c|}{$S 4.1: \kappa \lambda$} & \multicolumn{5}{|c|}{$S 4.2: \mu \nu$} \\
\hline cell & 1 & 2 & 3 & 4 & 5 & 1 & 2 & 3 & 4 & 5 \\
\hline number of & $p$ & $\frac{(p-1) p}{2}$ & $N p$ & $\frac{N p(p+1)}{2}$ & $N p$ & $p$ & $\frac{(p-1) p}{2}$ & $N p$ & $\frac{N p(p+1)}{2}$ & $N p$ \\
\hline sq.rt & - & - & - & - & - & - & - & - & - & - \\
\hline div. & 1 & - & - & - & 1 & 1 & - & - & - & 1 \\
\hline mult. & 8 & 4 & 6 & 5 & 1 & 5 & 3 & 5 & 4 & - \\
\hline i/o & 8 & 12 & 19 & 14 & 4 & 6 & 8 & 14 & 10 & 4 \\
\hline & \multicolumn{5}{|c|}{$S 4.3:$ Givens rotation } & & & & & \\
\hline cell & 1 & 2 & 3 & 4 & 5 & & & & & \\
\hline number of & $p$ & $\frac{(p-1) p}{2}$ & $N p$ & $\frac{N p(p+1)}{2}$ & $N p$ & & & & & \\
\hline sq.rt & 1 & - & - & - & - & & & & & \\
\hline div. & 1 & - & - & - & 1 & & & & & \\
\hline mult. & 4 & 4 & 5 & 5 & - & & & & & \\
\hline $\mathrm{i} / \mathrm{o}$ & 4 & 8 & 13 & 10 & 4 & & & & & \\
\hline
\end{tabular}

TABLE V

Minimum Required Delay between Two Consequent Sets of InPut Data

\begin{tabular}{|c|c|c|c|c|c|}
\hline & operations-per-cycle & $\begin{array}{c}\text { DSP96000 } \\
\text { (ns) }\end{array}$ & $\begin{array}{c}\text { IMS T800 } \\
\text { (ns) }\end{array}$ & $\begin{array}{c}\text { WEITEK } 3164 \\
\text { (ns) }\end{array}$ & $\begin{array}{c}\text { ADSP-3201/2 } \\
\text { (ns) }\end{array}$ \\
\hline S1.1 & $\max \{1$ div. +1 mult., 9 mult. $\}$ & 900 & 3150 & 1800 & 2700 \\
\hline $\mathrm{S} 1.2$ & 1 div. +5 mult. & 1020 & 2300 & 2700 & 3675 \\
\hline $\mathrm{S} 1.3$ & 1 sq.rt. +1 div. +4 mult. & 1810 & 4500 & 5300 & 7175 \\
\hline S2.1 & 8 mult. & 800 & 2800 & 1600 & 2400 \\
\hline $\mathrm{S} 2.2$ & 1 div. +5 mult. & 1020 & 2300 & 2700 & 3675 \\
\hline S2.3 & 1 sq.rt. +1 div. +4 mult. & 1810 & 4500 & 5300 & 7175 \\
\hline S3.1 & 1 div, +9 mult. & 1420 & 3700 & 3500 & 4875 \\
\hline S3.2 & 1 div. +6 mult. & 1120 & 2650 & 2900 & 3975 \\
\hline S3.3 & 1 sq.rt. +1 div. +5 mult. & 1810 & 4500 & 5300 & 7175 \\
\hline S4.1 & 1 div. +8 mult. & 1320 & 3350 & 3300 & 4575 \\
\hline $\mathrm{S} 4.2$ & 1 div. +5 mult. & 1020 & 2300 & 2700 & 3675 \\
\hline S4.3 & 1 sq.rt. +1 div. +4 mult. & 1810 & 4500 & 5300 & 7175 \\
\hline
\end{tabular}

Consequently, the numerical stability of the $\mathrm{QRD}$ architectures based on these algorithms can be questioned. Furthermore, a crucial piece of information in the circuit design is the wordlength, that is the number of bits per word required to ensure correct operations of the algorithm without overflow. At the same time, the wordlength has large impact on the complexity and the speed of the hardware implementation. In this section, we address issues on stability, error bounds and lower bounds for the wordlength by means of dynamic range analysis. We' focus on the algorithm for RLS optimal residual extraction based on a $\kappa \lambda$ Rotation. The dynamic range of the variables involved in the other newly introduced algorithms can be computed in a similar way.

In [13], Liu et al. study the dynamic range of the QRD-RLS algorithm that utilizes the standard Givens rotation. This study is based on the fact that the rotation parameters generated by the boundary cells of the systolic QRD-RLS structure eventually reach a quasi-steady-state regardless of the input data statistics, provided that the forgetting factor $\beta$ is close to one. A worst case analysis of the steady state dynamic range reveals the bound [13]

$$
\begin{array}{r}
\lim _{n \rightarrow \infty}\left|r_{i j}(n)\right| \leq \frac{(2 \beta)^{i-1}}{\sqrt{1-\beta^{2}}}\left|x_{\max }\right| \triangleq \mathcal{R}_{i}^{r}, \\
j=i, i+1, \cdots, p+1
\end{array}
$$

for the contents of the processing elements of the $i$ th row in the systolic structure, $i=1,2, \cdots, p$, where $\left|x_{\max }\right|$ is the largest value in the input data. Similarly, at the steady state the output of the $i$ th row $x_{j}^{(i)}, j=i, i+1, \cdots, p+1$ is bounded by [13]

$$
\begin{aligned}
\lim _{n \rightarrow \infty}\left|x_{j}^{(i)}(n)\right| & \leq(2 \beta)^{i-1}\left|x_{\max }\right| \triangleq \mathcal{R}_{i}^{x}, \\
j & =i+1, i+2, \cdots, p+1 .
\end{aligned}
$$

Furthermore, the optimal residual $e_{R L S}$ is bounded by [13]

$$
\lim _{n \rightarrow \infty}\left|e_{R L S}(n)\right| \leq(2 \beta)^{p-1}\left|x_{\max }\right| \triangleq \mathcal{R}_{i}^{\text {err }} .
$$

The latter is a BIBO stability result that applies also for the QRD-RLS algorithm based on a $\kappa \lambda$ Rotation. Nevertheless, the internal stability is not guaranteed. More concretely, the terms involved in the QRD-RLS algorithm may not be upper bounded.

In view of the internal stability problem, a proper choice of the parameters $\kappa$ and $\lambda$ should be made. A correct choice will compensate for the denormalization of the type

$$
r_{i j}^{\prime}=\frac{1}{\sqrt{l_{i}^{\prime}}} a_{i j}^{\prime}, \quad x_{j}^{(i)}=\frac{1}{\sqrt{l_{q}^{(i)}}} b_{j}^{(i)}
$$

where $l_{i}^{\prime}$ and $l_{q}^{(i)}$ are given in (22) and (23), respectively. The terms $\kappa_{i}^{2}$ and $\lambda_{i}^{2}$ in (22) and (23) can be used as shift operators by choosing

$$
\kappa_{i}=2^{-\rho_{i}} \text { and } \lambda_{i}=2^{-\tau_{i}}, \quad i=1,2, \cdots, p
$$




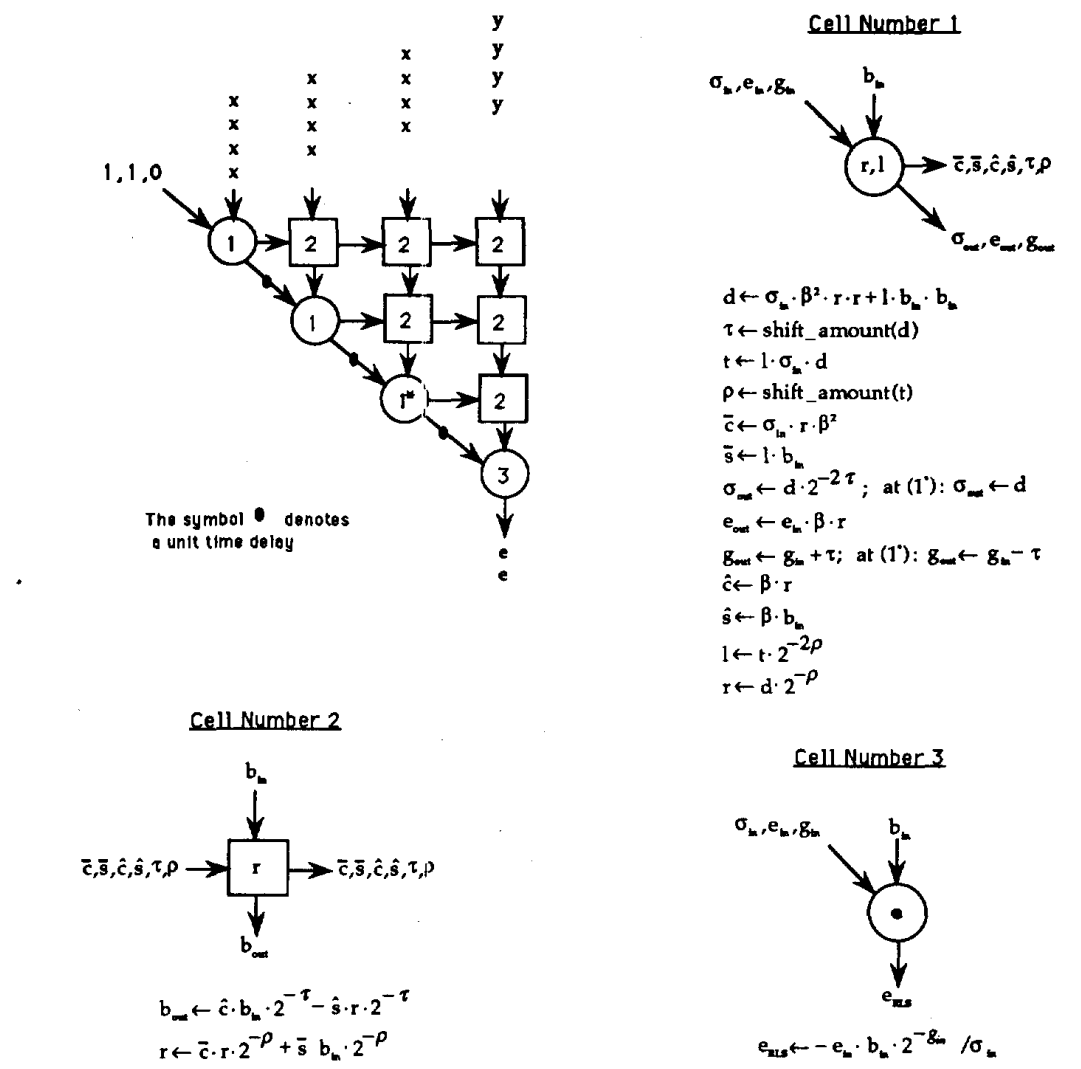

Fig. 6. Systolic array that computes the RLS optimal residual based on the scaled square root free and division free $\Re$ otation.

where $\rho_{i}$ and $\tau_{i}$ take integer values. For instance, in (23), if $\tau_{i}>0$ the effect of $\lambda_{i}^{2}$ on $\left(l_{q}^{(i-1)} \beta^{2} a_{i i}^{2}+l_{i} b_{i}^{(i-1)^{2}}\right.$ will be a right shift of $2 \tau_{i}$ bits. We can ensure that

$$
0.5 \leq l_{i}^{\prime}<2 \text { and } 0.5 \leq l_{q}^{(i)}<2, \quad i=1,2, \cdots, p
$$

by forcing the most significant bit (MSB) of the binary representation to be either at position $2^{0}$ or $2^{-1}$ after the shift operation. This normalization task has been introdiced in [1] and further used in [4]. It can be described in analytic terms by the expression

$$
\begin{aligned}
& \text { shift_amount(unnormalized_quantity) } \\
& \left.=\left\lfloor\left\{\log _{2} \text { (unnormalized_quantity) }+1\right\} / 2\right\rfloor\right\rfloor
\end{aligned}
$$

and it can be implemented very easily in hardware

In the sequel, we consider the $\kappa \lambda$ Rotation by choosing

$$
\begin{aligned}
\rho_{i} & =\text { shift_amount }\left[l_{i} l_{q}^{(i-1)}\left(l_{q}^{(i-1)} \beta^{2} a_{i i}^{2}+l_{i} b_{i}^{(i-1)^{2}}\right)\right] \\
\tau_{i} & =\text { shift_amount }\left[\left(l_{q}^{(i-1)} \beta^{2} a_{i i}^{2}+l_{i} b_{i}^{(i-1)^{2}}\right)\right]
\end{aligned}
$$

for $i=1,2, \cdots, p$ [4]. Note that (46) along with (44) should precede (22)-(25) in the rotation algorithm. In conformity to [1] and [4] we will refer to the resulting rotation algorithm with the name scaled rotation.

The systolic array that implements the QRD-RLS algorithm for the optimal residual extraction is depicted in Fig. 6. A comparison of this systolic array with the one in Fig. 2 is summarized by the following points: The boundary cells generate the shift quantities $\rho$ and $\tau$ associated with the parameters $\kappa$ and $\lambda$, respectively, and they communicate them horizontally with the internal cells. This yields two additional links for the boundary cells and four additional ones for the internal cells. In the dynamic range study that follows, we show that the number of bits these links occupy is close to the logarithm of the number of bits required by the rest of the links. The boundary cells are also responsible for computing the quantities $\prod_{i=1}^{p} \beta a_{i i}$ and $\prod_{i=1}^{p-1} \lambda_{i}$ in (26). In this case, $\lambda_{i}$ is an exponential term according to (44), so the above product can be computed as the running sum of the exponents

$$
g_{i}=\sum_{k=1}^{i} \tau_{k}, \quad i=1,2, \cdots, p-1
$$

yielding an additional adder for the boundary cells. Finally, as far as the boundary cells are concerned, we observe that the cell at position $(p, p)$ of the systolic array is not identical to the rest of the boundary cells. This is a direct consequence of (26). On the other hand, the shift operators constitute the only overhead of the internal and the output cells compared with the corresponding ones in Fig. 2. Overall, the computational complexity (in terms of operator counts) is slightly higher than that of the systolic array with $\kappa=\lambda=1$.

Let us focus now on the dynamic range of the variables in the systolic array. By solving (43) for $a_{i j}^{\prime}$ and using (41) and 
(45) one can compute an upper bound for $a_{i j}$ at the steady state, thus one can specify the dynamic range of the $i$ th row cell content. A similar result can be obtained for the output of the $i$ th row by using (42), (43) and (45). The results are summarized by the following Lemma:

Lemma 5.1: The steady state dynamic range of the cell content $\mathcal{R}_{i}^{a}$ and output range $\mathcal{R}_{i}^{b}$ in the $i$ th row are given by

$$
\begin{aligned}
& \lim _{n \rightarrow \infty}\left|a_{i j}(n)\right| \leq \mathcal{R}_{i}^{a} \triangleq \sqrt{2} \mathcal{R}_{i}^{r} \text { and } \\
& \lim _{n \rightarrow \infty}\left|b_{j}^{(i)}(n)\right| \leq \mathcal{R}_{i}^{b \triangleq \sqrt{2}} \mathcal{R}_{i}^{x}
\end{aligned}
$$

respectively.

The lower bounds in the wordlength come as a direct consequence of Lemma 4: The wordlength of the cell content $\beta_{i}^{a}$ and output $\beta_{i}^{b}$ in the $i$ th row must be lower bounded by

$$
\beta_{i}^{a} \geq\left\lceil\beta_{i}^{r}+0.5\right\rceil \text { and } \beta_{i}^{b} \geq\left\lceil\beta_{i}^{x}+0.5\right\rceil
$$

respectively, where $\beta_{i}^{r}=\left\lceil\log _{2} \mathcal{R}_{i}^{r}\right\rceil$ and $\beta_{i}^{x}=\left\lceil\log _{2} \mathcal{R}_{i}^{x}\right\rceil$ are the corresponding wordlength lower bounds for the QRD-RLS implementation based on the standard Givens rotation.

The parameters $\kappa_{i}, \lambda_{i}$ are communicated via their exponents $\rho_{i}$ and $\tau_{i}$. The dynamic ranges of these exponents are given by Lemma 5 which is proved in the Appendix.

Lemma 5.2: The steady state dynamic range of the terms $\rho_{i}$ and $\tau_{i}$ at the $i$ th row $\mathcal{R}_{i}^{\rho}$ and $\mathcal{R}_{i}^{\tau}$ are given by

$$
\begin{aligned}
\lim _{n \rightarrow \infty} \rho_{i} \leq \mathcal{R}_{i}^{\rho} \triangleq \beta_{i}^{a}+2.5 \\
\lim _{n \rightarrow \infty} \tau_{i} \leq \mathcal{R}_{i}^{\tau} \triangleq \beta_{i}^{a}+1.5
\end{aligned}
$$

respectively ${ }^{7}$, if both $\rho_{i}$ and $\tau_{i}$ are nonnegative.

Obviously, if both $\rho_{i}$ and $\tau_{i}$ take negative values, (50) will also satisfy. But there is no guarantee in its dynamic bound. Notice that taking negative value means a left shift. Uncontrolled arbitrary left shift may end up losing the MSB, an equivalence of overflow. Thus, it will be wise to also limit the magnitudes of negative $\rho_{i}$ and $\tau_{i}$ to the bounds in (50), i.e.

$$
\begin{aligned}
& \lim _{n \rightarrow \infty}\left|\rho_{i}\right| \leq \mathcal{R}_{i}^{\rho} \\
& \lim _{n \rightarrow \infty}\left|\tau_{i}\right| \leq \mathcal{R}_{i}^{\tau}
\end{aligned}
$$

Consequently, the lower bounds on the wordlength $\beta_{i}^{\rho}$ and $\beta_{i}^{\tau}$ of $\rho_{i}$ and $\tau_{i}$ are

$$
\begin{aligned}
& \beta_{i}^{\rho} \geq\left\lceil\log \left(\beta_{i}^{a}+2.5\right)\right\rceil \text { and } \\
& \beta_{i}^{\tau} \geq\left\lceil\log \left(\beta_{i}^{a}+1.5\right)\right\rceil
\end{aligned}
$$

respectively.

For the computation of the optimal residual the boundary cells need to evaluate both the running product $e_{i}=$ $\prod_{k=1}^{i} \beta a_{k k}$ and the running sum in (47). The dynamic ranges for these terms are given by the following Lemma:

\footnotetext{
${ }^{7}$ For the sake of simplicity in notation we have dropped the time parameter $n$ from the expression in the limit argument.
}

Lemma 5.3: The steady state dynamic range of the terms $e_{i}$ and $g_{i}$ at the $i$ th row $\mathcal{R}_{i}^{e}$ and $\mathcal{R}_{i}^{g}$ are given by

$$
\begin{aligned}
& \lim _{n \rightarrow \infty}\left|e_{i}\right| \leq \mathcal{R}_{i}^{e} \triangleq \prod_{k=1}^{i} \mathcal{R}_{k}^{a} \\
& \lim _{n \rightarrow \infty}\left|g_{i}\right| \leq \mathcal{R}_{i}^{g} \triangleq i \beta_{1}^{a}+\frac{i(i+2)}{2}
\end{aligned}
$$

respectively.

The proof is given in the Appendix. With simple algebraic manipulations one can show that the corresponding lower bounds on wordlength $\beta_{i}^{e}$ and $\beta_{i}^{g}$ of $e_{i}$ and $g_{i}$ are

$$
\begin{aligned}
& \beta_{i}^{e} \geq \sum_{k=1}^{i} \beta_{k}^{a} \text { and } \\
& \beta_{i}^{g} \geq \max \left\{\left\lceil\log \beta_{1}^{a}+\log i+1\right\rceil,\lceil\log i+\log (i+2)\rceil\right\}
\end{aligned}
$$

respectively.

Finally, consider the coefficients defined as

$$
\begin{array}{ll}
\bar{c}_{i}=l_{q}^{(i-1)} \beta^{2} a_{i i} & \bar{s}_{i}=l_{i} b_{i}^{(i-1)} \\
\hat{c}_{i}=\beta a_{i i} & \hat{s}_{i}=\beta b_{i}^{(i-1)},
\end{array}
$$

that describe the information exchanged by the remaining horizontal links in the systolic array (cf. Fig. 6). One can easily show that the steady state dynamic range of these coefficients, denoted by $\mathcal{R}_{i}^{\bar{c}}, \mathcal{R}_{i}^{\bar{s}}, \mathcal{R}_{i}^{\hat{c}}$ and $\mathcal{R}_{i}^{\hat{s}}$, respectively are

$$
\begin{gathered}
\lim _{n \rightarrow \infty}\left|\bar{c}_{i}\right| \leq \mathcal{R}_{i}^{\bar{c}} \triangleq 2 \mathcal{R}_{i}^{a} \\
\lim _{n \rightarrow \infty}\left|\bar{s}_{i}\right| \leq \mathcal{R}_{i}^{\bar{s}} \triangleq 2 \mathcal{R}_{i}^{b} \\
\lim _{n \rightarrow \infty}\left|\hat{c}_{i}\right| \leq \mathcal{R}_{i}^{\hat{c}} \triangleq \mathcal{R}_{i}^{a} \\
\lim _{n \rightarrow \infty}\left|\hat{s}_{i}\right| \leq \mathcal{R}_{i}^{\hat{s} \triangleq \mathcal{R}_{i}^{b} .}
\end{gathered}
$$

The implied wordlength lower bounds are $\beta_{i}^{\bar{c}} \geq \beta_{i}^{a}+1$, $\beta_{i}^{\bar{s}} \geq \beta_{i}^{b}+1, \beta_{i}^{\hat{c}} \geq \beta_{i}^{a}$ and $\beta_{i}^{\hat{s}} \geq \beta_{i}^{b}$, respectively.

In summary, (45), (48), (50), (53), and (55) show that all the internal parameters are bounded and therefore the algorithm is stable. Furthermore, the lower bounds on the wordlength provide the guidelines for an inexpensive, functionally correct realization.

The error bound of the whole QRD to a given matrix $A \in \Re^{m \times n}$ due to floating point operations is given by [1], [4]

$$
\|\delta A\| \leq \tau(m+n-3)(1+\tau)^{m+n-4}\|A\|+O\left(\epsilon^{2}\right),
$$

where $\tau$ is the upper bound and $\epsilon$ is the largest number such that $1+\epsilon$ is computed as 1 . If (44) and (45) are satisfied, for $\kappa=\lambda=1$, then it follows that $\tau=6.5 \epsilon$ [4]. This is fairly close to the standard Givens rotation which has $\tau=6.0 \epsilon[4]$.

\section{CONCLUSION}

We introduced the parametric $\kappa \lambda \Re$ Rotation, which is a square-root-free and division-free algorithm, and showed that the parametric $\kappa \lambda$ Rotation describes a subset of the $\mu \nu$ Rotation algorithms [8]. We then derived novel architectures based on the $\kappa \lambda$ Rotation for $\kappa=\lambda=1$ and made a 
comparative study with the standard Givens rotation and the $\mu \nu \Re$ Rataion with $\mu=\nu=1$. Finally, a dynamic range study is pursued. It is observed that considerable improvements can be obtained for the implementation of some QRD-based algorithms.

We pointed out the tradeoffs between the architectures based on the above Rotations. Our analysis su:gests the following decision rule for selecting between the aritectures that are based on the $\mu \nu$ Rotation and the $\kappa \lambda \Re$ otation: Use the $\mu \nu$ Rotation - based architectures, with $\mu=$ $\nu=1$, for the constrained minimization problems and the $\kappa \lambda \Re$ otation - based architectures, with $\kappa=\lambda=1$, for the unconstrained minimization problems. Table $\mathrm{V}$ shows the benchmark comparisons of different ilgorithms using different DSP processors and it confirms the properties claimed in this paper.

A number of obscure points relevant to the realization of the QRD-RLS and the QRD-CRLS algorithms are clari ied. Some systolic structures that are described in this pape: are very promising, since they require less computational complexity (in various aspects) from the structures known tc date and they make the VLSI implementation easier.

\section{APPENDIX}

Proof of Lemma 3.1: First, we derive some equations that will be used in the course of the optimal residual computation.

If we solve (24), case $i=j=1$, for $l_{q} \beta^{2} a_{11}^{2}-l_{1} b_{1}^{2}$ and substitute in (22) we get

$$
l_{1}^{\prime}=l_{1} l_{q} \frac{a_{11}^{\prime}}{\kappa_{1}} \kappa_{1}^{2}
$$

and therefore

$$
\frac{l_{1}^{\prime}}{l_{1}}=l_{q} a_{11}^{\prime} \kappa_{1}
$$

If we solve (24), case $j=i$, for $l_{q}^{(i-1)} \beta^{2} a_{i i}^{2}+l_{i} b^{i-1)^{2}}$ and substitute in (23) we get

$$
l_{q}^{(i)}=\frac{\lambda_{i}^{2} a_{i i}^{\prime}}{\kappa_{i}} .
$$

If we substitute the same expression in (22) we get.

$$
l_{i}^{\prime}=l_{i} l_{q}^{(i-1)} a_{i i}^{\prime} \kappa_{i}
$$

(58), and solve for $l_{i}^{\prime} / l_{i}$ to obtain

$$
\frac{l_{i}^{\prime}}{l_{i}}=\frac{\lambda_{i-1}^{2} \kappa_{i}}{\kappa_{i-1}} a_{i-1, i-1}^{\prime} a_{i i}^{\prime} .
$$

If we solve (22) for $l_{q}^{(i-1)} \beta^{2} a_{i i}^{2}+l_{i} b_{i}^{(i-1)^{2}}$ and substitute in (23) we get

$$
l_{q}^{(i)}=\frac{\lambda_{i}^{2}}{\kappa_{i}^{2}} \frac{l_{i}^{\prime}}{l_{i}} \frac{1}{l_{q}^{(i-1)}} .
$$

Also, we note that (4) implies that

$$
c_{i}=\beta r_{i i} / r_{i i}^{\prime}
$$

and by substituting (9) we obtain

$$
c_{i}=\frac{\beta a_{i i}}{a_{i i}^{\prime}} \sqrt{\frac{l_{i}^{\prime}}{l_{i}}} \quad i=1,2, \cdots, p .
$$

Similarly, from (4) and (9), we get

$$
s_{i}=\frac{b_{i}^{(i-1)}}{a_{i i}^{\prime}} \sqrt{\frac{l_{i}^{\prime}}{l_{q}^{(i-1)}}} \quad i=1,2, \cdots, p .
$$

The optimal residual for the RLS problem is [6]

$$
e_{R L S}\left(t_{n}\right)=-\left(\prod_{k=1}^{p} c_{k}\right) v\left(t_{n}\right) .
$$

The expressions in (20) and (19) imply

$$
v\left(t_{n}\right)=\frac{1}{\sqrt{l_{q}^{(p)}}} b_{p+1}^{(p)} .
$$

If we substitute the above expressions of $v\left(t_{n}\right)$ and $c_{i}$ in (63) we obtain

$$
e_{R L S}\left(t_{n}\right)=-\prod_{i=1}^{p}\left(\frac{\beta a_{i i}}{a_{i i}^{\prime}} \sqrt{\frac{l_{i}^{\prime}}{l_{i}}}\right) \frac{1}{\sqrt{l_{q}^{(p)}}} b_{p+1}^{(p)} .
$$

From (60) we get

$$
\begin{aligned}
& l_{q}^{(p)}=\frac{\lambda_{p}^{2}}{\kappa_{p}^{2}} \frac{l_{p}^{\prime}}{l_{p}} \frac{1}{l_{q}^{(p-1)}}=\frac{\lambda_{p}^{2}}{\kappa_{p}^{2}} \frac{l_{p}^{\prime}}{l_{p}} \frac{\kappa_{p-1}^{2}}{\lambda_{p-1}^{2}} \frac{l_{p-1}}{l_{p-1}^{\prime}} l_{q}^{(p-2)} \\
& =\left\{\begin{array}{cc}
\prod_{j=1}^{k}\left(\frac{\lambda_{2 j}^{2}}{\kappa_{2 j}^{2}} \frac{l_{2 j}^{\prime}}{l_{2 j}} \frac{\kappa_{2 j-1}^{2}}{\lambda_{2 j}^{2}-1} \frac{l_{2 j-1}}{l_{2 j-1}^{\prime}}\right) l_{q}, & p=2 k \\
\prod_{j=1}^{k-1}\left(\frac{\kappa_{2 j}^{2}}{\lambda_{2 j}^{2}} \frac{l_{2 j}}{l_{2 j}^{\prime}} \frac{\lambda_{2 j-1}^{2}}{\kappa_{2 j-1}^{2}} \frac{l_{2 j-1}^{\prime}}{l_{2 j-1}}\right) \frac{\lambda_{p}^{2}}{\kappa_{p}^{2}} \frac{l_{p}^{\prime}}{l_{p}} \frac{1}{l_{q}}, & p=2 k-1
\end{array} .\right.
\end{aligned}
$$

Thus, from (64) and (65), for the case of $p=2 k$, we have the first equation at the top of the next page. By doing the appropriate term cancelations and by substituting the expressions of $l_{i}^{\prime} / l_{i}, i=1,2, \cdots, 2 k$ from (57) and (59) we obtain the expression (26) for the optimal residual. Similarly, for the case of $p=2 k-1$, from (64) and (65) we obtain the second equation at the top of the next page and by substituting (59), we get (26)

Proof of Lemma 3.2: The question is whether we can avoid the division in the evaluation of the residual. Obviously we should $\lambda_{p} a_{p p}^{\prime}$ or

$$
\lambda_{p}=\kappa_{p} / a_{p p}^{\prime}
$$

holds. But, from (24), for $j=i$, we get

$$
\kappa_{p} / a_{p p}^{\prime}=\frac{1}{l_{q}^{(p-1)} \beta^{2} a_{p p}^{2}+l_{p} b_{p}^{(p-1)^{2}}} .
$$

Therefore, if we choose to avoid the division operation in the expression of the residual, we will need to perform another division in order to evaluate the parameter $\lambda_{p}$. 


$$
e_{R L S}\left(t_{n}\right)=-\prod_{j=1}^{k}\left(\frac{\beta a_{2 j, 2 j}}{a_{2 j, 2 j}^{\prime}} \sqrt{\frac{l_{2 j}^{\prime}}{l_{2 j}}} \frac{\beta a_{2 j-1,2 j-1}}{a_{2 j-1,2 j-1}^{\prime}} \sqrt{\frac{l_{2 j-1}^{\prime}}{l_{2 j-1}}}\left(\frac{\lambda_{2 j}^{2}}{\kappa_{2 j}^{2}} \frac{l_{2 j}^{\prime}}{l_{2 j}} \frac{\kappa_{2 j-1}^{2}}{\lambda_{2 j-1}^{2}} \frac{l_{2 j-1}}{l_{2 j-1}^{\prime}}\right)^{-\frac{1}{2}}\right) \cdot \frac{1}{\sqrt{l_{q}}} \cdot b_{p+1}^{(p)}
$$

$$
e_{R L S}\left(t_{n}\right)=-\prod_{j=1}^{k-1}\left(\frac{\beta a_{2 j, 2 j}}{a_{2 j, 2 j}^{\prime}} \sqrt{\frac{l_{2 j}^{\prime}}{l_{2 j}}} \frac{\beta a_{2 j-1,2 j-1}}{a_{2 j-1,2 j-1}^{\prime}} \sqrt{\frac{l_{2 j-1}^{\prime}}{l_{2 j-1}}}\left(\frac{\lambda_{2 j-1}^{2}}{\kappa_{2 j-1}^{2}} \frac{l_{2 j-1}^{\prime}}{l_{2 j-1}} \frac{\kappa_{2 j}^{2}}{\lambda_{2 j}^{2}} \frac{l_{2 j}}{l_{2 j}^{\prime}}\right)^{-\frac{1}{2}}\right) \cdot \frac{\beta a_{p p}}{a_{p p}^{\prime}} \sqrt{\frac{l_{p}^{\prime}}{l_{p}}} \sqrt{\frac{\kappa_{p}^{2} l_{p} l_{q}}{\lambda_{p}^{2} l_{p}^{\prime}}} b_{p+1}^{(p)}
$$

Proof of Lemma 5.2: From (45) and the fact that $0<$ $\beta<1$ we get

$$
l_{q}^{(i-1)} \beta^{2} a_{i i}^{2}+l_{i} b_{i}^{(i-1)^{2}}<2 a_{i i}(n)^{2}+2 b_{i}^{(i-1)^{2}} .
$$

Consequently, at the steady state we have

$$
\lim _{n \rightarrow \infty}\left|l_{q}^{(i-1)} \beta^{2} a_{i i}^{2}+l_{i} b_{i}^{(i-1)^{2}}\right|<2\left(\mathcal{R}_{i}^{a}\right)^{2}+2\left(\mathcal{R}_{i}^{b}\right)^{2} .
$$

Also, (41), (42), and (48) imply that $\mathcal{R}_{i}^{a}>\mathcal{R}_{i}^{b}$. Therefore, we obtain the bound

$$
\lim _{n \rightarrow \infty}\left|l_{q}^{(i-1)} \beta^{2} a_{i i}^{2}+l_{i} b_{i}^{(i-1)^{2}}\right|<4\left(\mathcal{R}_{i}^{a}\right)^{2}
$$

and by utilizing (23) and the fact that $l_{q}^{(i)} \geq 0.5$ we get

$$
\begin{aligned}
\lim _{n \rightarrow \infty}\left|\lambda_{i}^{-2}\right| & \leq 2 \lim _{n \rightarrow \infty}\left|l_{q}^{(i-1)} \beta^{2} a_{i i}^{2}+l_{i} b_{i}^{(i-1)^{2}}\right| \\
& <8\left(\mathcal{R}_{i}^{a}\right)^{2} .
\end{aligned}
$$

By substituting the expression $\lambda_{i}=2^{-\tau_{i}}$, using (66) and solving the resulting inequality for $\tau_{i}$

$$
\lim _{n \rightarrow \infty} \tau_{i} \leq \log \mathcal{R}_{i}^{a}+1.5
$$

if $\tau_{i}$ is nonnegative. The expression for the dynamic range of $\tau_{i}$ in (50) is a direct consequence of the above inequality.

Similarly, for the computation of the dynamic range of the term $\rho_{i}$ first one can prove that

$$
\lim _{n \rightarrow \infty}\left|l_{i} l_{q}^{(i)}\left(l_{q}^{(i-1)} \beta^{2} a_{i i}^{2}+l_{i} b_{i}^{(i-1)^{2}}\right)\right|<16\left(\mathcal{R}_{i}^{a}\right)^{2}
$$

and then compute an upper bound for $\rho_{i}$ at the steady state based on (22), (44) and the fact that $l_{i}^{\prime} \geq 0.5$.

Proof of Lemma 5.3: Since $0<\beta<1$, for the term $e_{i}$ we have

$$
\lim _{n \rightarrow \infty}\left|e_{i}\right|=\beta^{i} \prod_{k=1}^{i} \lim _{n \rightarrow \infty}\left|a_{k k}\right| \leq \prod_{k=1}^{i} \mathcal{R}_{k}^{a} .
$$

Similarly, for the term $g_{i}$ we have

$$
\lim _{n \rightarrow \infty}\left|g_{i}\right|=\sum_{k=1}^{i} \lim _{n \rightarrow \infty}\left|\tau_{k}\right|
$$

and from (50)

$$
\lim _{n \rightarrow \infty}\left|g_{i}\right| \leq \sum_{k=1}^{i} \mathcal{R}_{k}^{\tau}=\sum_{k=1}^{i}\left(\beta_{k}^{a}+1.5\right) .
$$

Equation (41) implies that the wordlength for the variable $r$ should satisfy the inequality

$$
\beta_{i}^{r} \geq\lceil(i-1)(1+\log \beta)+C\rceil
$$

where $C$ is constant with respect to $i$. Since $\beta<1$, it is sufficient to have

$$
\beta_{i}^{r} \geq\lceil i-1+C\rceil
$$

or

$$
\beta_{i}^{r} \geq i-1+\beta_{1}^{r} .
$$

A similar formula can be derived for the wordlength of the contents of the the array that utilizes the scaled rotation, based on (49) and (68). More specifically, we have

$$
\beta_{i}^{a} \geq \beta_{1}^{a}+i-1 \text {. }
$$

From this inequality and (67) we get

$$
\lim _{n \rightarrow \infty}\left|g_{i}\right| \leq i \beta_{i}^{a}+\frac{i(i-1)}{2}+1.5 i .
$$

The dynamic range expression in (53) follows directly.

\section{REFERENCES}

[1] J. L. Barlow and I. C. F. Ipsen, "Scaled Givens rotations for the solution of linear least squares problems on systolic arrays," SIAM J. Sci., Statist. Comput., vol. 8, no. 5, pp. 716-733, Sept. 1987.

[2] W. M. Gentleman, "Least squares computations by Givens transformations without square roots," J. Inst. Math. Applicat., vol. 12, pp. 329-336, 1973.

[3] W. M. Gentleman and H. T. Kung, "Matrix triangularization by systolic arrays," in Proc. SPIE 298, Real-Time Signal Processing IV, pp. 19-26, 1981 .

[4] J. Gotze and U. Schwiegelshohn, "A square root and division free Givens rotation for solving least squares problems on systolic arrays," SIAM J. Sci., Statist. Comput., vol. 12, no. 4, pp. 800-807, July 1991.

[5] S. Hammarling, "A note on modifications to the Givens plane rotation," J. Inst. Math. Applicat., vol. 13, pp. 215-218, 1974.

[6] S. Haykin, Adaptive Filter Theory, 2nd ed. Englewood Cliffs, NJ: Prentice-Hall, 1991.

[7] S. F. Hsieh, K. J. R. Liu, and K. Yao, "Dual-state systolic architectures for up/downdating RLS adaptive filtering," IEEE Trans. Circuits, Syst. II, vol. 39, no. 6, pp. 382-385, June 1992 .

[8] — "A unified approach for QRD-based recursive least-squares estimation without square roots," IEEE Trans. Signal Processing, vol. 41, no. 3, pp. 1405-1409, March 1993.

[9] S. Kalson and K. Yao, "Systolic array processing for order and time recursive generalized least-squares estimation," in Proc. SPIE 564, RealTime Signal Processing VIII, 1985, pp. 28-38. 
[10] F. Ling, "Efficient least squares lattice algorithm based on Givens rotations with systolic array implementation," IEEE Trans. Signal Processing, vol. 39, pp. 1541-1551, July 1991.

[11] F. Ling, D. Manolakis, and J. G. Proakis, "A recursive modified GramSchmidt algorithm for least-squares estimation, IEEE Truns. Acoust., Speech, Signal Processing, vol. ASSP-34, no. 4, pp. 829-836, Aug. 1986.

[12] K. J. R. Liu, S. F. Hsieh, and K. Yao, "Systolic block Housholder transformation for RLS algorithm with two-level pipelined imp ementation," IEEE Trans. Signal Processing, vol. 40, no. 4, pp. 946-95i;, Apr. 1992.

[13] K. J. R. Liu, S. F. Hsieh, K. Yao, and C. T. chiu, "Dy ramic range, stability and fault-tolerant capability of finite precision i?LS systolic array based on Givens rotation," IEEE Trans. Circuits, S Sist., vol. 38, no. 6 , pp. 625-636, June 1991 .

[14] J. G. McWhirter, "Recursive least-squares minimization us ing a systolic array," in Proc. SPIE, Real Time Signal Processing VI, vo. 431, 1983 pp. $105-112$.

[15] J. G. McWhirter and T. J. Shepherd, "Systolic array frocessor for MVDR beamforming," in IEE Proc., pt. F, vol. 136, no. ?., Apr. 1989 pp. $75-80$.

[16] I. K. Proulder, J. G. McWhirter, and T. J. Shepherd, "The QRD-based least squares lattice algorithm: Some computer simulation * using finite wordlength," in Proc. IEEE ISCAS (New Orleans, LA), May 1990, pp. 258-261

[17] T. J. Shepherd, J. G. McWirter, and J. E. Hudson, "Parallel weight extraction from a systolic adaptive beamforming," Math. Signell Processing II, 1990.

[18] R. W. Stewart, R. Chapman, and T. S. Durrani, "Arith netic implementation of the Givens QR tiarray," in Proc. IEEE/ICAS\$P, 1989, pp. V-2405-2408.

[19] C. F. T. Tang, K. J. R. Liu, and S. Tretter, "Optimal weight extraction for adaptive beamforming using systolic arrays," IEEE Trins. Aerosp. Electron. Syst., vol. 30, no. 2, pp. 367-385, Apr. 1994.

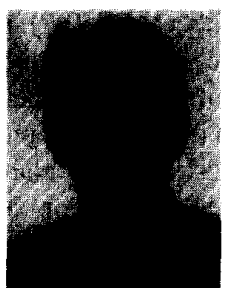

E. N. Frantzeskakis (S'90-M'93) was born in Athens, Greece, in 1965 . He received the B.S. degree in computer engineering and information science from the University of Patras, Greece, in 1988 , and the M.S. and Ph.D. degrees in electrical engineering from the University of Maryland, College Park, in 1990 and 1993, respectively.

From August 1988 to August 1993, ie worked at the Systems Research Center, UMCP, as a Graduate Research Assistant. Currently, he is with INTRACOM, S.A. Athens, Greece. His resecrch interests include VLSI architectures for real-time digital signal processing. HDTV, and adaptive filtering.

Dr. Frantzeskakis is a member of the Technical Chamber of (ireece.

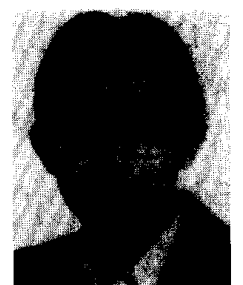

K. J. R. Liu (S'86-M'90-SM'93) received the B.S. degree in electrical engineering from National Taiwan University in 1983, the M.S.E. degree in electrical engineering and computer science from the University of Michigan, Ann Arbor, in 1987, and the Ph.D. degree in electrical engineering from the University of California, Los Angeles, in 1990.

Since 1990, he has been an Assistant Professor of Electrical Engineering Department and Institute for Systems Research, University of Maryland, College Park. His research interests span all aspects of high performance computational signal processing including parallel and distributed processing, fast algorithm, VLSI, and concurrent architecture, with application to image/video, radar/sonar, communications, and medical and biomedical technology.

Dr. Liu received the IEEE Signal Processing Society's 1993 Senior Award and the 1994 National Science Foundation Young Investigator Award. He was awarded the George Corcoran Award for outstanding contributions to electrical engineering education at the University of Maryland. He has also received many other awards including the Research Initiation Award from the National Science Foundation, the President Research Partnership from the University of Michigan, and the University Fellowship and the Hortense Fishbaugh Memorial Scholarship from the Taiwanese-American Foundation. He is an Associate Editor of the IEEE TRANSACTIONS ON SIGNAL PROCESSING and a member of the VLSI Signal Processing Technical Committee of the IEEE Signal Processing Society. 